\title{
Superior Mesenteric Artery thrombosis in COVID-19 pneumonia: An underestimated diagnosis - First case report in Asia
}

\section{Dr Sunaina Tejpal Karna ( $\nabla$ drtejpal@gmail.com )}

All India Institute of Medical Sciences, Bhopal https://orcid.org/0000-0002-4200-5948

\section{Dr Rajesh Panda}

All India Institute of Medical Sciences, Bhopal

\section{Dr Ajeet Pratap Maurya}

All India Institute of Medical Sciences, Bhopal

\section{Dr Saurab Saigal}

All India Institute of Medical Sciences, Bhopal

\section{Case Report}

Keywords: Superior mesenteric artery (SMA) thrombosis, Mesenteric ischemia, COVID-19 pneumonia

Posted Date: July 29th, 2020

DOI: https://doi.org/10.21203/rs.3.rs-31156/v1

License: (c) (i) This work is licensed under a Creative Commons Attribution 4.0 International License. Read Full License

Version of Record: A version of this preprint was published at Indian Journal of Surgery on October 19th, 2020. See the published version at https://doi.org/10.1007/s12262-020-02638-5. 


\section{Abstract}

The COVID-19 disease caused by novel Coronavirus was first reported in Wuhan, China in December 2019 with $5 \%$ patients having severe lung injury. Though this disease primarily presents as a lower respiratory tract infection, multiple digestive manifestations have been reported which are often overlooked. The present case report describes the unusual progression of COVID-19 disease from pneumonia to a procoagulant state leading to superior mesenteric artery thrombosis and subsequent gut ischemia necessitating emergency laparotomy. Coagulopathy in COVID-19 is due to an imbalance in the coagulation homeostasis with increase in thrombocytes in contrast to thrombocytopenia documented in cases of dissemination intravascular coagulation and sepsis. Early recognition of abdominal symptoms, diagnosis of pathology and timely surgical intervention may definitely improve outcome. In the management of any patient with COVID-19 disease, we advocate a comprehensive integrated approach with early recognition of digestive symptoms and their timely intervention which should run parallel to the respiratory management.

\section{Introduction}

COVID-19 Pandemic has affected the whole world with 4,289,084 confirmed cases, and 288,346 deaths as of 12thMay 2020 [1]. Although primary presentation is Pneumonia, there are multiple digestive manifestations [2]. The present case report describes the unique progression of the COVID-19 infection from pneumonia to superior mesenteric artery thrombosis and subsequent gut ischemia necessitating emergency laparotomy.

\section{Case Presentation}

On 28th April 2020, a 61 year old diabetic, hypertensive female presented with respiratory distress to our Institute, a dedicated COVID-19 Centre. With pulse110bpm, Blood pressure 140/78 mmHg and Saturation on room air $78 \%$, supplemental oxygen was given by face mask. A paired nasopharyngeal and oral swab was sent for RT- PCR to rule out COVID-19 infection. She was shifted to covid suspect ICU. Her routine blood investigations were sent (Table 1) and bedside chest Xray was done which showed pneumonia pattern in the peripheral and basal region. Treatment was started with high flow nasal oxygen(HFNO), Cefoperazone-Sulbactam, prophylactic enoxaparin, pantoprazole, vitamin $\mathrm{C}$ and zinc as per institutional protocol. She showed symptomatic improvement with SpO2 of $95 \%$.

On fourth day of admission, she developed diffuse abdominal pain with distention. Emergent contrast CT scan abdomen showed thrombosis of distal superior mesenteric artery (SMA) with dilated jejunoileal loops and normal enhancing bowel wall. Therapeutic dose of unfractionated heparin 5000 units intravenous followed by maintenance dose of 1000 Units/hr infusion was administered with Ecosprin, Clopidogrel, still thrombocytosis persisted. A repeat paired swab for RT-PCR sent with suspicion of COVID19 pneumonia and hyperthrombotic state was positive. There was symptomatic relief in abdominal distension and pain with bowel movements, so sips of water followed by liquid diet was started. 
On tenth day of admission, patient complained of acute abdominal pain and distention with two episodes of feculent vomiting. Emergency exploratory laparotomy was planned after correction of coagulopathy. Intra- operative findings were gangrenous distal ileum $60 \mathrm{~cm}$ in length $30 \mathrm{~cm}$ proximal to ileocecal junction. Mesentery was thickened and inflamed, no bleeding from distal mesenteric vessels but pulsation present in proximal superior mesenteric artery. Small perforation present in gangrenous bowel, rest of small and large bowel were healthy. Resection of gangrenous bowel and loop ileostomy done. Postoperative course was complicated by septic shock and acute renal failure. Despite maximal supportive care, she succumbed to the illness 36 hours after surgery.

\section{Discussion}

We report the first case of Superior Mesenteric Artery thrombosis in a patient with COVID-19 pneumonia in Asia. After favourable evolution of disease with anticoagulant therapy, there was mesenteric ischemia manifested by acute abdominal pain. Though surgical exploration and resection of gangrenous bowel was successful, patient succumbed to vasoplegic shock and multiorgan dysfunction syndrome.

In the course of COVID-19 disease, hypoxia, inflammatory mediators, thrombocythemia, immobilization, sepsis, liver injury secondary to ACE2 receptor expression may predispose to arterial and venous thrombosis [2,3]. In our case, there was serial increase in platelet levels with sustained elevated CRP levels (table1) suggesting reciprocity of thrombotic and inflammatory state.

Till now, only five cases of SMA thrombosis in COVID-19 positive patients are reported in the world till now (3,2,1 patient in Strasbourg, Paris and Italy, respectively) [4-6]. Of these, one patient with multiple comorbidities was managed conservatively but did not survive. In the 4 operated patients, only two(28 years female, 52 years male) survived while one (56 years male) was still on ventilatory support. Surgical procedures performed were: Jejunal resection with laparostomy followed by double jejunostomy and abdominal wall closure 2days later(28 year female), bowel resection and side to side stapled anastomosis (52 years old male). Microthrombi and inflammatory mediators were postulated to cause mesenteric ischemia. Though anticoagulation did improve disease evolution, sudden abdominal pain was noted in both cases, in spite of continuation of anticoagulation in first and after cessation of heparin post discharge in second case. CT Scan was diagnostic in both cases.

In Mesenteric ischemia, the time lag between onset of symptom to treatment is crucial for good outcome. The optimal time for intervention is initial 12 hours from symptom onset, when it is possible to perform vascular surgery effectively without requiring intestinal resection [7]. However, patients with COVID-19 often present late or treatment of respiratory symptoms is given precedence over abdominal symptoms [2]. Mucosal ischemia may further induce massive spread of virus from bowel epithelium leading to vasoplegic shock after surgery, as was noted in our case [8].

In management of any patient with COVID 19 disease, a holistic approach should be adopted with evaluation of digestive symptoms along with respiratory. SMA thrombosis should be suspected if patient has abdominal distension or pain with existing thrombocythemia with increased inflammatory markers. 
For diagnosis, Contrast enhanced CT Scan should be done and it may need to be repeated to see the progression of disease. Emergency laparotomy may have a favourable outcome if done immediately after onset of abdominal pain and before onset of any new organ failure.

\section{Declarations}

Funding: none

Conflict of interest: Authors declare no conflict of interest

Consent: Consent was given by the patient as well as her husband for publication of Case report and use of peri-operative data for research purposes.

\section{References}

1. Worldometer COVID-19 Data,12 may 2020,https://www.worldometers.info/coronavirus/coronavirusdeath-toll

2. Pan L, Mu M, Yang P, Sun Y, Wang R, Yan J, Li P, Hu B, Wang J, Hu C, Jin Y, Niu X, Ping R, Du Y, Li T, Xu G, Hu Q, Tu L (2020) Clinical Characteristics of COVID-19 Patients With Digestive Symptoms in Hubei, China: A Descriptive, Cross-Sectional, Multicenter Study. Am J Gastroenterol 115(5):766-773. doi: 14309/ajg.0000000000000620

3. Klok FA, Kruip MJHA, van der Meer NJM, Arbous MS, Gommers DAMPJ, Kant KM, Kaptein FHJ, van Paassen J, Stals MAM, Huisman MV, Endeman H (2020) Incidence of thrombotic complications in critically ill ICU patients with COVID-19. Thromb Res S0049-3848(20)30120-1. doi: 1016/j.thromres.2020.04.013

4. Beccara L, Pacioni C, Ponton S, Francavilla S, Cuzzoli A (2020) Arterial Mesenteric Thrombosis as a complication of SARS-CoV-2 Infection. European Journal of Case Reports in Internal Medicine 7(5). org/10.12890/2020_001690

5. de Barry O, Mekki A, Diffre C, Seror M, Hajjam ME, Carlier RY (2020) Arterial and venous abdominal thrombosis in a 79-year-old woman with COVID-19 pneumonia. Radiol Case Rep. doi:

10.1016/j.radcr.2020.04.055

6. Ignat M, Philouze G, Aussenac-Belle L, Faucher V, Collange O, Mutter D, Pessaux P (2020) Small bowel ischemia and SARS-CoV-2 infection: an underdiagnosed distinct clinical entity. Surg journal. doi: 1016/j.surg.2020.04.035.

7. Jagielski M, Piątkowski J, Jackowski Challenges Encountered during the Treatment of Acute Mesenteric Ischemia (2020) Gastroenterol Res Pract. doi: 10.1155/2020/5316849. 
8. Xiao F, Tang M, Zheng X, Liu Y, Li X, Shan H (2020) Evidence for Gastrointestinal Infection of SARSCoV-2. Gastroenterology;158(6):1831-1833.doi: 1053/j.gastro.2020.02.055.

\section{Tables}

TABLE-1 (Serial trend of blood investigations) : 1.Hb (Hemoglobin),2.TPC(Total platelet count), 3.TLC(Total leucocyte count), 4.NLR(Neutrophil to lymphocyte ratio), 5.CRP(C reactive protein), 6.Activated partial thromboplastin time, 7.Prothrombin time/International normalized ratio

\begin{tabular}{|l|l|l|l|l|l|l|}
\hline PARAMETERS & $28 / 4 / 20$ & $30 / 4 / 20$ & $3 / 5 / 20$ & $6 / 5 / 20$ & $8 / 5 / 20$ & $9 / 5 / 20$ \\
\hline Hb1 in gm/dl & 12.4 & 11.2 & 10.4 & 9.4 & 8.7 & 7.1 \\
\hline TPC2 in microlitre & $4,01,000$ & $4,64,000$ & $5,20,000$ & $5,74,000$ & $5,79,000$ & $3,11,000$ \\
\hline $\begin{array}{l}\text { TLC3 per cubic } \\
\text { millimeter }\end{array}$ & 21,300 & 17,000 & 11,600 & 12,000 & 18,200 & 23,000 \\
\hline NLR4 & 10.2 & 19.3 & 10.5 & 12.5 & 15 & 18.5 \\
\hline Monocyte & 10 & 8 & 9 & 5 & 4 & 5 \\
\hline CRP5 in mg/dl & 343 & 421.6 & 437 & 304 & 132 & 282 \\
\hline $\begin{array}{l}\text { Triglycerides in } \\
\text { mg/dl }\end{array}$ & 105 & 466 & 712 & 672 & 411 & 105 \\
\hline aPTT6 & & & 53 & 51.8 & 30.8 & 32.9 \\
\hline PT/INR7 & & & $20 / 1.8$ & $22 / 2.1$ & $21.3 / 2$ & $19 / 1.7$ \\
\hline Lactate mmol/l & 1.1 & 1.4 & 1.5 & 1.9 & 1.6 & 8.4 \\
\hline pH & 7.49 & 7.45 & 7.44 & 7.49 & 7.43 & 7.11 \\
\hline
\end{tabular}

\section{Figures}




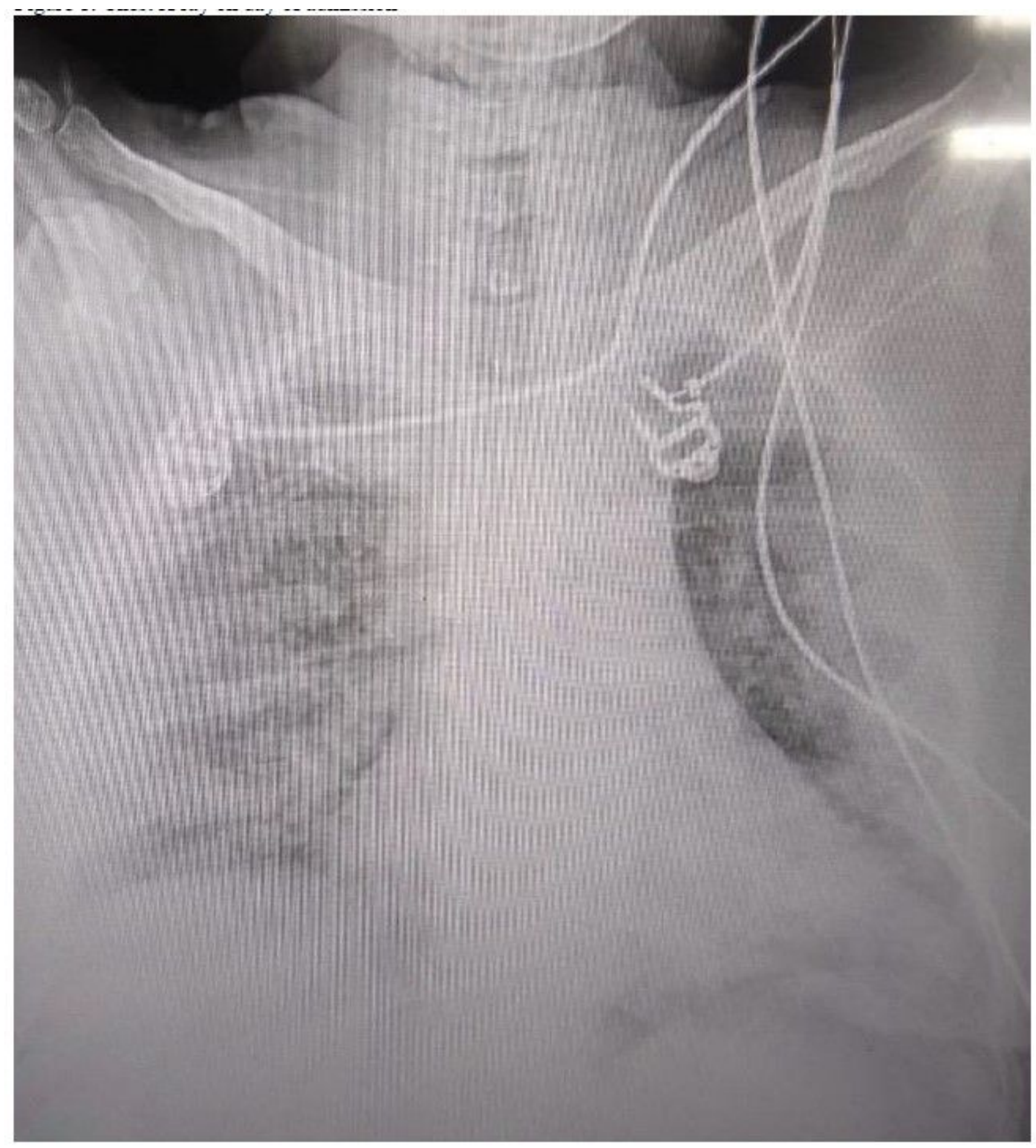

Figure 1

Chest $X$ ray on day of admission 

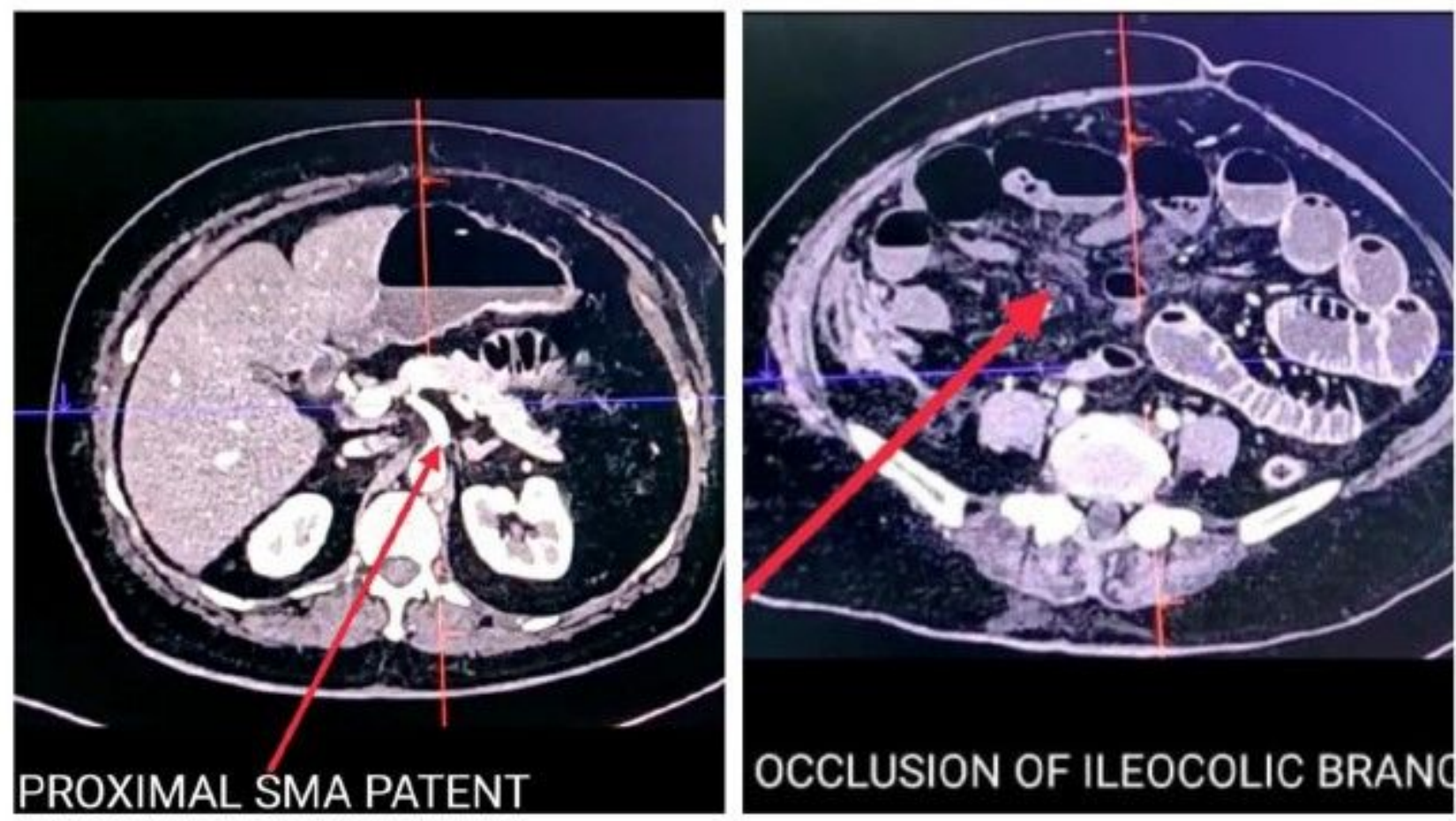

Figure 2

CT cut section showing patent proximal superior mesenteric artery with occlusion of distal ileocolic branch 